\title{
Gestión Territorial y Estrategia Política ${ }^{1}$
}

\author{
Cátia Wanderley Lubambo
}

Postdoctorada en Gestión Pública (FGV-SP) y Doctora en Sociología (UFPE).

Investigadora Titular de la Dirección de Investigaciones Sociales/Fundación Joaquim Nabuco-Recife. Docente en la Maestría Profesional en Gestión Pública para el Desarrollo del Nordeste, en la UFPE. Maestra en Desarrollo Urbano y Regional (UFPE).

Email: catia.lubambo@fundaj.gov.br

\section{RESUMEN}

Recepción: 2012-03-23

Aceptación: 2012-04-18

El texto presenta una discusión sobre la gestión del territorio como estrategia política, a partir de la experiencia reciente de Brasil con programas centrados en la participación social. Se comparan cuatro experiencias municipales en el ámbito de la gestión territorial de dos programas estatales: el Programa Gobierno en los Municipios, en el estado de Pernambuco, y el Proyecto Mi Sitio, en Santa Catarina. Llaman la atención la competitividad política local y la ausencia de capacidad institucional en los gobiernos subnacionales, como factores limitantes al éxito de las experiencias de acción pública descentralizada. Existe una referencia analítica a otros estudios que enfocan fórums regionales de programas territoriales y que suelen señalar resultados en esa dirección, tanto en el ámbito de los ministerios como de los gobiernos estaduales. El análisis muestra reflexiones sobre cómo factores político-institucionales pueden ser favorables a la actuación de fórums participativos y sugiere un abordaje que considere a los actores sociales locales en la definición del territorio, en contraposición a las concepciones formadas a partir de criterios sólo programáticos.

Palabras-claves: Territorio, Gestión Pública, Descentralización.

\section{ABSTRACT}

This paper presents a discussion of land management as a political strategy, based on the recent experience in Brazil with programs focused on social participation. It compares four municipal

\footnotetext{
1 Investigación financiada por el CNPq y la Fundación Joaquim Nabuco en Brasil.
} 
experiences in the área of territorial management of two state programs: the Government Program in the mnicicipalities, in the state of Penambuco, and Project My Site in Santa Catarina. It draws our attention the local policital competitiveness and lack of institutional capacity of the subnational governments as limiting factors to the success in the experiences of decentralized public action. There is an analytical references to the other studies focused on regional forums of territorial programs that often point results on that direction, at both of the ministries and the state governments. The analysis shows reflections on how political-institutional factors can be favorable to the performance of participatory forums and suggests and approach that considers the local stakeholders in defining the territory, as opposed to the conceptions formed from only programmatic criteria.

Key words: Territory, Public Management, Decentralization.

\section{INTRODUCCIÓN}

La reducida relevancia de los factores político-institucionales, observada en propuestas para acciones públicas en el territorio, causa preocupación. La cuestión de la regionalización ha sido tratada, principalmente, a partir de criterios técnicos y, sobre todo, económicos, de tal forma que la variable cultura política es simplemente anunciada como una dimensión explicativa de los fracasos; y no investigada con la debida profundidad analítica.

De modo casi unánime, la concepción de territorio es rápidamente comprendida como un proceso que envuelve decisiones, además de la implementación de acciones públicas, independientemente de fronteras adminis- trativas predefinidas por la estructura federativa $^{2}$. Sin embargo, aunque se comprenda -por un lado- la gestión territorial como un mecanismo de destino óptimo de recursos y -por otro- como un fórum de articulación de actores políticos, el territorio no suele ser visto como estrategia política, tanto en el ámbito electoral como en el ámbito de las políticas públicas. La reducción analítica de los factores político-institucionales, en niveles territoriales, acaba revelando lagunas de naturaleza política en propuestas de territorialización.

2 El concepto de territorio aquí adoptado se aproxima de aquel presentado por la Consejería de Desarrollo Territorial del Ministerio del Desarrollo Agrario: "un espacio fisico, geográficamente definido, generalmente continuo, comprendiendo la ciudad y el campo, caracterizado por criterios multidimensionales - tales como el ambiente, la economía, la sociedad, la cultura, la política y las instituciones -, y una población con grupos sociales relativamente distintos, que se relacionan interna y externamente por medio de procesos específicos, donde se puede distinguir uno o más elementos que indican identidad y cohesión social, cultural y territorial." (MDASDT, 2003) 
Con ese propósito, el estudio ha buscado investigar los factores que interfieren en la gestión territorial. Partiendo de la experiencia de dos programas de políticas subnacionales - el Programa Gobierno en el Estado, en Pernambuco, y el Proyecto Mi Sitio, en Santa Catarina -, creemos que la competitividad política local y la ausencia de capacidad institucional en los gobiernos subnacionales merecen mayor atención. Tales factores han creado -o mantenido- una red intrigante de intereses y se encuentran reflejados en las experiencias de acción pública descentralizada, independientemente del nivel de coordinación que ellas propongan. Estudios basados en investigaciones de campo, que enfocan los fórums regionales de varios programas territoriales, han señalado en esa dirección; tanto en el ámbito de los ministerios como de los gobiernos estaduales.

En el Ministerio de la Integración Regional, la ilustración se sitúa en el ámbito de la elaboración de la Política Nacional de Ordenamiento Territorial (PNOT), con el documento Directrices para Formulación de Políticas de Desarrollo Regional y de Ordenación del Territorio Brasileño, realizado por el Cedeplar/UFMG en 2004, para la Consejería de Políticas de Desarrollo Regional. En 2006, en el Ministerio de Desarrollo Agrario fue divulgado un estudio sobre territorios nacionales, ${ }^{3}$

3 PCT/SDT/MDA/IICA (2006). Desarrollo Sostenible y Territorialidad: identidades y tipologías. Informe Final. que más tarde sirvió de referencia para la elaboración del Programa Territorios de la Ciudadanía.

El Programa Territorios de la Ciudadanía, creado el 25 de febrero de 2008, consiste en una "estrategia de desarrollo regional sostenible y garantía de derechos sociales volcada a las regiones del país que más necesitan, con el objetivo de llevar el desarrollo económico y universalizar los programas básicos de ciudadanía [...] En cada territorio, un Consejo Territorial compuesto por las tres esferas gubernamentales y por la sociedad, determinará un plan de desarrollo y una agenda pactada de acciones [...] Fueron definidos conjuntos de municipios unidos por los mismos rasgos económicos y ambientales que tengan identidad y cohesión social, cultural y geográfica”. Actualmente el Programa contempla 60 territorios distribuidos por todo Brasil, y en cada estado federativo hay por lo menos un Territorio de la Ciudadanía.

En una oportunidad más reciente, durante el Seminario Internacional de Planeamiento Territorial en Brasil, promovido por el Ministerio de Planeamiento, Presupuesto y Gestión, en noviembre de 2008 en Brasília, fue presentado un Estudio de la Dimensión Territorial del País, con la indicación de los vectores estratégicos de desarrollo. Además fue propues-

4 http://www.mda.gov.br/portal/index/show/index/cod/1816/ codInterno/16264. Investigación realizada en 22/09/2008. 


\section{GESTIÓN TERRITORIAL Y PARTICIPACIÓN POLÍTICA}

\subsection{Cuestiones Centrales}

A pesar de la multiplicidad de conceptos, al definir territorio prevalece un cierto consenso sobre la necesidad de separación de las barreras político-administrativas definidas en el federalismo brasileño. El concepto puede, entonces, ser aplicado a una unidad menor que la extensión del municipio, igual a la del municipio, mayor que la del municipio, igual a la de un grupo o de partes de municipios en estados distintos, etc. De esa manera, una iniciativa de gestión territorial en el país impone una negociación en torno de la reconstrucción del territorio preexistente, mediante la aprobación del proyecto de ley enviado a la Cámara de Concejales, a la Asamblea Estadual o al Congreso Nacional, en función del abarcamiento del caso. Una serie de problemas previsibles y no previsibles, inherentes al proceso político administrativo -que acaba derivando de esa iniciativa una vez que intereses de más de un órgano federativo-, estarán envueltos.

Esta discusión se basa en el concepto de las arenas políticas, según lo discutido por Löwi (LÖWI, 1964 y 1985), sobre los principales factores determinantes de la estructuración de las arenas, así como sobre la tipología de políticas. En su trabajo, Löwi divide las políticas (policy arenas, en el original) en cuatro tipos fundamentales: las políticas distributivas, redistributivas, regulatorias y constitutivas. Dentro de esa perspectiva cada una de las arenas políticas acarrea rasgos y comportamientos propios por parte de los actores ${ }^{5}$. En el caso de los pactos territoriales se trata de una arena propia de las políticas constitutivas. Las políticas constitutivas son políticas que modifican las reglas del juego; cuestiones relativas al diseño o a la estructura de funcionamiento del gobierno, que reflejan la distribución de poder y autoridad entre organizaciones de la burocracia gubernamental. Con eso, esas políticas generan estructuras de incentivos propios, distintos de las estructuras previamente existentes. De esa manera, la generación de una nueva política constitutiva, como la de gestión territorial, lleva a la definición o al cambio de las reglas del juego político. O sea, los actores que definirán los cambios de ese tipo de arena son, en ese caso específico, órganos federativos; una vez que el ordenamiento territorial vaya a modificar la estructura de destino y transferencia de recursos del Gobierno Federal para estados y municipios y, por lo tanto, reorganizará los intereses políticos en esos territorios.

5 Todavía de acuerdo con Lowi, los actores políticos envueltos no necesariamente presentan comportamientos invariables con relación a su envolvimiento y su posición en la política. La referencia es más direccionada a comportamientos típicos: el de grupos de interés, el de clientela, el de partidos políticos y el de elite tecnocrática. Algunos autores también llaman a las políticas constitutivas politicas estructuradoras; en este texto, usaremos ambos los términos. 


\section{GESTIÓN TERRITORIAL Y PARTICIPACIÓN POLÍTICA}

\subsection{Cuestiones Centrales}

A pesar de la multiplicidad de conceptos, al definir territorio prevalece un cierto consenso sobre la necesidad de separación de las barreras político-administrativas definidas en el federalismo brasileño. El concepto puede, entonces, ser aplicado a una unidad menor que la extensión del municipio, igual a la del municipio, mayor que la del municipio, igual a la de un grupo o de partes de municipios en estados distintos, etc. De esa manera, una iniciativa de gestión territorial en el país impone una negociación en torno de la reconstrucción del territorio preexistente, mediante la aprobación del proyecto de ley enviado a la Cámara de Concejales, a la Asamblea Estadual o al Congreso Nacional, en función del abarcamiento del caso. Una serie de problemas previsibles y no previsibles, inherentes al proceso político administrativo -que acaba derivando de esa iniciativa una vez que intereses de más de un órgano federativo-, estarán envueltos.

Esta discusión se basa en el concepto de las arenas políticas, según lo discutido por Löwi (LÖWI, 1964 y 1985), sobre los principales factores determinantes de la estructuración de las arenas, así como sobre la tipología de políticas. En su trabajo, Löwi divide las políticas (policy arenas, en el original) en cuatro tipos fundamentales: las políticas distributivas, redistributivas, regulatorias y constitutivas. Dentro de esa perspectiva cada una de las arenas políticas acarrea rasgos y comportamientos propios por parte de los actores 5 . En el caso de los pactos territoriales se trata de una arena propia de las políticas constitutivas. Las políticas constitutivas son políticas que modifican las reglas del juego; cuestiones relativas al diseño o a la estructura de funcionamiento del gobierno, que reflejan la distribución de poder y autoridad entre organizaciones de la burocracia gubernamental. Con eso, esas políticas generan estructuras de incentivos propios, distintos de las estructuras previamente existentes. De esa manera, la generación de una nueva política constitutiva, como la de gestión territorial, lleva a la definición o al cambio de las reglas del juego político. O sea, los actores que definirán los cambios de ese tipo de arena son, en ese caso específico, órganos federativos; una vez que el ordenamiento territorial vaya a modificar la estructura de destino y transferencia de recursos del Gobierno Federal para estados y municipios y, por lo tanto, reorganizará los intereses políticos en esos territorios.

5 Todavía de acuerdo con Lowi, los actores políticos envueltos no necesariamente presentan comportamientos invariables con relación a su envolvimiento y su posición en la política. La referencia es más direccionada a comportamientos típicos: el de grupos de interés, el de clientela, el de partidos políticos y el de elite tecnocrática. Algunos autores también llaman a las políticas constitutivas politicas estructuradoras; en este texto, usaremos ambos los términos. 
La mayor parte del debate público en Brasil suele abordar las transformaciones institucionales en el plan de la descentralización de la gestión, que ocurre por dos vías principales. En primer lugar, por la ampliación de la participación en las decisiones públicas, a través de mecanismos de consulta, que envuelven a la población directamente, mediante la institución de fórums y plenarias locales ${ }^{6}$. En segundo lugar, por el fortalecimiento de los mecanismos de control de acompañamiento de gestión territorial, mediante la creación de instancias de deliberación y consulta, ${ }^{7}$ que agrupan representantes de los intereses directamente envueltos, como también de entidades de la sociedad civil, proveedores de servicios y clientes.

De acuerdo con la sugerencia del propio debate teórico, (LUBAMBO, COELHO, MELO, 2005 y ARRETCHE et alli, 2006), dificultades mayores al empoderamiento surgen en la misma medida en que se expresa la resistencia de la sociedad a la participación. Esa resistencia se presenta principalmente como resultado de una herencia cívica desfavorable, que se hace todavía presente en muchas regiones y localidades del país, como en los pe-

6 Los ejemplos más destacados en esa dirección han sido las experiencias de presupuesto participativo.

7 Es posible afirmar que ha ocurrido un verdadero choque institucional en la última década, señalado por la creación de centenas; en algunos casos millares, de consejos vinculados a los planes locales en las áreas de niños y adolescentes, desarrollo urbano, educación, desarrollo rural, medio ambiente, salud y asistencia social. Ese hecho contribuye para que el tema reciba espacio importante en la literatura. Ver GOHN (2001); CARVALHO (1995); COELHO y NOBRE (2004), entre otros. queños municipios del Norte y del Nordeste brasileño. Por un lado, se debe a una reducida credibilidad en el Estado, debilitada por una serie de gobiernos descomprometidos con el bienestar de la población y; por otro lado, a una ausencia de experiencias locales de asociativismo (bajo capital social). Son evidentes los límites de las burocracias acostumbradas a los antiguos modelos y resistentes a estrategias de reingeniería institucional por parte del Estado. De modo similar son reconocidos límites contenidos en la representación y en la participación popular, intermediada por asociaciones de cualquier especie, sobre todo por aquellas procedentes de cambios institucionales, como los modelos programáticos con nítida orientación gubernamental. Independientemente de la discusión sobre la eficacia de esas instituciones/asociaciones, la participación/representación de la población requiere de preparación para enfrentar los problemas más simples de la acción colectiva.

Los consejos de representación de la sociedad civil se han convertido en un componente esencial del diseño institucional de las políticas públicas actuales en Brasil. Los consejos se difundieron, de tal manera, que es rara una política pública cuyo mecanismo regulador de operación no cuente con al menos un consejo, cuya existencia se deba a una exigencia de la legislación. Para las más diversas políticas y en los tres niveles de gobierno, se han 
convertido en una especie de elemento constitutivo de su diseño institucional.

Como resultado de la generalización de la exigencia de los consejos (ABRAMOVAY, 2001; MELO, 2003), también se observa la emergencia de instancias de esa naturaleza para las políticas y los programas de desarrollo territorial. En pocas palabras, la inclusión de mecanismos de participación política para las experiencias de gestión territorial no ha surgido por cualquier manifestación espontánea de la sociedad civil. Éstas surgieron, principalmente, como requisitos de programas financiados, en su mayoría, por agencias internacionales y por el Gobierno Federal. La exigencia de la participación de la comunidad beneficiaria en la financiación y la manutención del proyecto, está entre las recomendaciones de muchos de los programas de desarrollo, como supuesto de que producirán los incentivos necesarios a la generación de capital social y, por consecuencia, desarrollo económico local.

Aunque se reconozcan relativas ganancias como fruto de estrategias de participación en las decisiones de destino de recursos de los programas, inspiradas en la teoría del capital social, una gestión territorial envuelve una política de naturaleza distributiva (LOWI, 1964). Es decir, se asignan beneficios de modo desagregado para distintas regiones y localidades y, por su condición participativa, representados por actores sociales también diversos. La identificación de elementos indicativos de cómo articular la acción de las instancias colegiadas para la gestión territorial, -de manera más integrada y cooperada en los recortes municipales, estadual y federal-, muestra una contribución a la consolidación y al perfeccionamiento de las prácticas asociadas a ese tipo de planeamiento y a la ejecución de programas de desarrollo.

Este es un análisis que busca focalizar los elementos y las condiciones que interfieren y favorecen la articulación, en diversos niveles, entre los fórums y consejos creados entre ellos, y los agentes responsables por la distribución de los beneficios, por programas que anuncian el desarrollo como resultado de la gestión territorial.

Como objetos de estudio serán consideradas cuatro experiencias municipales para un análisis comparativo, en el ámbito de la gestión territorial de dos programas estaduales: el Programa Gobierno en los Municipios, en Pernambuco y el Programa de Descentralización, en Santa Catarina.

El Programa Gobierno en los Municipios fue establecido en el estado de Pernambuco en 1999 y fue considerado, entre el conjunto de experiencias participativas que el país vive, 
como una novedad institucional. Éste estaba claramente orientado por la idea de que la sociedad puede imprimir una lógica más democrática en la definición de las prioridades y en el destino de los recursos públicos, estando "más próxima del Estado". Esa expectativa inicial se ha ampliado para la construcción de un modelo de gestión territorial, por el cual se proyectó la colecta de demandas específicas y la negociación sobre el ordenamiento de varias propuestas sectoriales, directamente con los grupos sociales, en cada una de las doce Regiones de Desarrollo del Estado. (Ver LUBAMBO, C. y COELHO, D. (2005)).

El Programa de Descentralización, en Santa Catarina, es una experiencia todavía más reciente. Éste programa fue implantado a partir del año 2003, basándose en los supuestos de descentralización de la gestión del estado y de la participación de la sociedad en el desarrollo del territorio. La idea central es que la descentralización del gobierno sea capaz de promover simultáneamente el desarrollo regional y construir un ambiente de cooperación y gobernabilidad, creando un círculo virtuoso y sinérgico de participación de varios sectores de la sociedad civil. El modelo básico ha propuesto la creación de los Consejos de Desarrollo Regional, vinculados a las Regiones de Desarrollo (30), con las Consejerías de Desarrollo Regional, creadas para mediar las demandas locales y el Gobierno Esta- dual. (GOBIERNO DE SANTA CATARINA. Disponible en: http://www.sc.gov.br/ Acceso en 28 de enero de 2008).

Aunque el reducido tiempo de implantación de los programas no favorezca afirmaciones totalmente conclusivas, el análisis aquí hecho sobre la experiencia de gestión participativa, con vistas a la gestión territorial, sugiere avances en la dirección del aprendizaje institucional. Pero también, muchos obstáculos para la efectiva implantación de un modelo experimental de gestión, basado en órganos que se superpongan a las estructuras políticas preexistentes. Hay una agenda para investigaciones próximas que se enfocan en el proceso de participación política mediante la existencia de otros mecanismos no formales, poco explícitos e inductores de la participación de los ciudadanos en la formulación e implantación de algunas acciones públicas específicas.

Entre esos mecanismos, las coaliciones electorales y los apoyos locales (municipales y territoriales) figuran como importantes y definidores de la competitividad política. Con tal abordaje, el estudio hace referencia a una línea de investigación propuesta por Ames (AMES, 1995 y 2003), en la que el autor analiza las condiciones políticas municipales para intentar explicar al comportamiento de los actores políticos y construir inferencias sobre los efectos de las propuestas de regiona- 
lización/territorialización, en la distribución/ concentración del voto. Sobre todo cuando se alían las motivaciones de control y accountability constitutivas de aquellas propuestas de gestión participativas. En resumen, los factores político-institucionales reordenados con la interferencia de la población, en niveles territoriales, empiezan a tener una relevancia más expresiva, considerando sobre todo, los actores políticos locales.

La aglomeración de municipios en territorios/regiones, según los programas referidos, crea nuevas condiciones importantes para el análisis político-institucional de la participación: a. aglomeración de ciudadanos electores de un mismo territorio (de "dominio" del parlamentar) o de dos o más municipios (de “dominio" de más alcaldes); b. la variable apoyo al gobernador es decisiva para la definición del territorio.

Por una hipótesis preliminar, hay fuertes indicios de que el establecimiento de los programas en cada uno de los estados y en los periodos respectivos, ha ejercido una influencia gradual en el cuadro político de apoyo a la primera y a la segunda gestión consecutiva de cada gobernador - Jarbas Vasconcelos en Pernambuco y Luís Henrique da Silveira en Santa Catarina-. Al parecer, la alianza partidaria se fortalece como una variable fundamental para garantizar la adhesión de las élites locales al programa. En los dos estados, los dos gobiernos fueron electos por medio de amplia coalición partidaria.

Con respecto a la competición política en la base municipal, históricamente, los diputados de esos partidos son individualmente más dominantes en sus bases electorales. En sus principales municipios, ellos tienden a quedarse con proporciones más altas de la votación total local. Generalmente son bien votados en municipios geográficamente contiguos y raras veces disputan la preferencia de los electores. Ese control oligárquico, representado por la concentración electoral, reduce no solamente el número de novatos en la política, como también el número de partidos competentes. Es decir, son padrones muy próximos de competición política.

\section{LAS VARIABLES ANALÍTICAS EN LAS LOCALIDADES DEL ESTUDIO - PERNAMBUCO Y SANTA CATARINA}

Este trabajo tiene como objeto de estudio el proceso de actuación de las instancias participativas de ámbito territorial en las regiones de análisis. Por hipótesis, las variables comúnmente referentes a la experiencia asociativa existente en los municipios, a los mecanismos de toma de decisión en el interior de los consejos específicos y a la articulación institucional engendrada para el tratamiento de las acciones 
públicas estarían mediadas por las variables competitividad local y apoyo de los políticos locales.

Como objeto de estudio, elemento para el análisis de la cuestión territorial, fue elegido el Consejo Municipal de Desarrollo Rural (CMDR), actuante en cada municipio de Pernambuco. En el estado de Santa Catarina fueron inclusas la Asociación de los Municipios de la Región Serrana (Amures) y la Consejería de Desarrollo Regional, ambas con sede en el municipio de Lages. La inclusión de las instancias complementarias para el caso de Santa Catarina, responden al modelo creado por la descentralización del estado, a partir del cual fueron constituidas Consejerías de Desarrollo Regional vinculadas a las Regiones de Desarrollo (30), al lado de los Consejos de Desarrollo Regional, creados para mediar las demandas locales y el Gobierno Estadual ${ }^{8}$.

Por opción analítica, fue observada la tradición asociativa en torno de proyectos de integración regional y de las condiciones socioeconómicas de los municipios seleccionados. Para las primeras condiciones, fue investigada la situación de los municipios, con base en la tipología propuesta por la Consejería de Desarrollo Regional del Ministerio de la Integración Nacional, que divide las microrregiones entre las de Alta Rienda, Dinámica,

8 GOBIERNO DE SANTA CATARINA. Disponible en: http://www. sc.gov.br/ Acceso en 28 de enero de 2008.
Estancada y de Baja Rienda9.

En el Cuadro 1 es posible observar que la muestra del número relativo de municipios de Pernambuco, diagnosticados como de baja rienda o en situación caracterizada como de estancamiento, es más elevado que el del correlato en Santa Catarina, incluso en relación al del resto del país. La gran concentración de territorios con bajo grado de desarrollo socioeconómico está expresada por el peso de un cuarto de los municipios del estado, contra menos de un $13 \%$ en Brasil y $0 \%$ en el estado de Santa Catarina. En ese estado, más de un $80 \%$ son considerados municipios de alta rienda; al contrario de Pernambuco, donde solamente $4,32 \%$ se encuadran en esa categoría.

Cuadro 1. Situación de los Municipios de Brasil, Pernambuco y Santa Catarina con Relación a la Dinámica de las Microrregiones

\begin{tabular}{|l|r|r|r|}
\hline $\begin{array}{c}\text { Categorías de } \\
\text { la Dinámica } \\
\text { Económica }\end{array}$ & Brasil & $\begin{array}{c}\text { Pernam- } \\
\text { buco }\end{array}$ & $\begin{array}{r}\text { Santa } \\
\text { Catarina }\end{array}$ \\
\hline Baja Rienda & 12,80 & 25,95 & 0,00 \\
\hline Estancada & 41,20 & 47,03 & 19,55 \\
\hline Dinámica & 17,13 & 22,16 & 0,00 \\
\hline Alta Rienda & 27,84 & 4,32 & 80,55 \\
\hline Total & 100,00 & 100,00 & 100,00 \\
\hline
\end{tabular}

Fuente: www.integracao.gov.br. Acceso en 28/04/2008.

9 Esta clasificación fue presentada con base en la tipología propuesta por la Consejería de Desarrollo Regional del Ministerio de la Integración Nacional, que divide las microrregiones entre las de Alta Rienda, Dinámica, Estancada y de Baja Rienda. En el texto, los términos región y territorio son utilizados indistintamente, una vez que hay una parte significativa de gestores que predican la integración de las políticas supramunicipales o supraestaduales en un único eje institucional. Cf. Ministerio de la Integración Nacional - Consejería de Políticas de Desarrollo Regional. 
Cuadro 2 - Población, Tasa de Urbanización e Índice de Desarrollo Humano en los Municipios Estudiados

\begin{tabular}{|l|c|c|c|}
\hline \multicolumn{1}{|c|}{ Municipios } & Población & $\begin{array}{c}\text { Tasa de Urbaniza- } \\
\text { ción }\end{array}$ & IDH \\
\hline Águas Belas PE) & $36.641 \mathrm{hab}$ & $54,00 \%$ & 0,53 \\
\hline Paranatama (PE) & $10.348 \mathrm{hab}$ & $16,00 \%$ & 0,56 \\
\hline Cerro Negro (SC) & $4.098 \mathrm{hab}$ & $16,94 \%$ & 0,69 \\
\hline Campo Belo do Sul (SC) & 8.051hab & $55,15 \%$ & 0,69 \\
\hline
\end{tabular}

Fuente: IBGE. Censo Demográfico 2000

La investigación ha tomado como universo de estudio dos regiones consideradas poco dinámicas en relación a otras regiones del Estado. En el estado de Pernambuco fue estudiada la región que se define por los 11 municipios contiguos de menor IDH del Agreste Meridional y Sertão do Moxotó $^{10}$, especialmente los municipios: Águas Belas y Paranatama. En el estado de Santa Catarina fue seleccionada la Região Serrana (serranía) de Lages, y los municipios elegidos fueron: Cerro Negro y Campo Belo do Sul1' ${ }^{11}$ Para los dos casos hubo una coincidencia entre la región elegida y el 10 Los municipios de ese territorio estadual son: Águas Belas, Caetés, Iati, Inajá, Ibimirim, Manari, Paranatama, Itaíba, Terezinha y Tupanatinga.

11 La investigación de campo fue realizada en dos momentos: 1. Pernambuco, en agosto de 2008, mediante la realización de entrevistas con los consejeros de desarrollo rural de los municipios de Águas Belas y de Paranatama y de entrevistas con los gestores Márcia Aguiar y Wédson Galindo, en Recife y con el coordinador regional para el Desarrollo Rural Ricardo Jucá; y los técnicos sociales Ricardo Oliveira, José Borges, Carla Moema, Josélia Assumpção y Charles Borges; 2. Santa Catarina, en septiembre de 2008, donde fueron realizadas entrevistas con Cristóvam Cardoso y Lori da Silveira, en la SDR Lages; Jocelito Matos - consejero regional, representante del municipio de Campo Belo; Jamerson Furtado - consejero regional, representante del municipio de Cerro Negro y, en Florianópolis, el consultor del PNUD Valério Turnes, la ex-secretaria ejecutiva de la Consejería de Planeamiento del Estado de Santa Catarina Anita Pires y el secretario ejecutivo de la Amures Gilsoni Albino. territorio definido por el MDA. ${ }^{12}$ La elección de los municipios, en el ámbito de esas regiones, se debe también a la evidencia de bajos niveles de IDH. Sin embargo, los municipios de la región de la Serra Catarinense ostenten IDHs más elevados que los del estado de Pernambuco. Ésta es “la más 'pobre' y 'retrasada' de todas las regiones" del estado. Ver MUNARIM (2000).

A continuación aparecen algunos datos que caracterizan el perfil de cada municipio estudiado.

Como son recurrentes análisis que articulan el grado de dinámica económica de los $\mathrm{Mu}$ nicipios y la capacidad de gestión para el de-

\footnotetext{
12 Los territorios definidos por el Ministerio de Desarrollo Agrario (MDA) son formados por 20 municipios en Pernambuco - Águas Belas, Angelim, Bom Conselho, Buíque, Caetés, Capoeiras,Garanhuns, Iati, Ibirajuba, Ibimirim, Inajá, Itaíba, Manari, Paranatama, Pedra, Saloá, São Bento do Una, Terezinha, Tupanatinga, Venturosa - y por 19 municipios en el estado de Santa Catarina: Anita Garibaldi, Bocaina do Sul, Bom Jardim da Serra, Bom Retiro, Campo Belo do Sul, Capão Alto, Cerro Negro, Correia Pinto, Lages, Otacílio Costa, Painel, Palmeira, Ponte Alta, Rio Rufino, São Joaquim, São José do Cerrito, Urubici y Urupema.
} 
sarrollo regional en una correlación inversa, los datos existentes para Pernambuco y Santa Catarina nos llevan a suposiciones en torno de la existencia de una mayor dificultad en la implantación de políticas territoriales, en aquel estado nordestino, en relación al último, perteneciente a la región Sur.

En esa misma dirección, se ha supuesto que para definir la variable como tradición de asociativismo municipal, las condiciones en la región Sur del país ya se presentan más favorables. Empíricamente la tradición de asociativismo municipal en Santa Catarina puede ser comprobada desde la década de 1960, con el movimiento municipal de Santa Catarina. En los años ochenta, surgió la Fecam (Federación Catarinense de los Municipios); en cuyos inicios fue creada con el nombre de Federación Catarinense de las Asociaciones Municipales, lo que realza el significado del número de entidades regionales de asociación de municipios. En 2008, la Fecam contaba con 284 municipios afiliados, de los 293 municipios pertenecientes a SC. Fueron las asociaciones de municipios coordinadas por la Fecam quienes dieron el impulso para la creación de los fórums de desarrollo en cada una de sus áreas territoriales (CÔRTES, 2006). En Pernambuco, la presencia existe, pero ocurre de modo poco sistemático. Entre algunos intentos de asociación municipal, pueden ser citadas la formación de un Fórum en la Mata Sur. Otros intentos fueron constituir consorcios municipales en el Agreste Central, (en torno de acciones de saneamiento y construcción de aterros sanitarios); y aún en el Submedio São Francisco, (en favor de acciones para instalación de centros de distribución y comercialización de productos).

¿Pero por qué estas condiciones tan divergentes de dinámica económica, capacidad de gestión para el desarrollo regional y tradición de asociativismo entre dos regiones se han vuelto secundarias, al mismo tiempo que otras condiciones más favorables indujeron a la experimentación de programas de territorialización y descentralización? ¿Cuáles fueron estas condiciones? En busca de esas respuestas fueron analizados los principales elementos que componen la dinámica político-institucional en los casos considerados. ${ }^{13}$

\subsection{El Caso de Pernambuco ${ }^{14}$}

Los indicadores asociados a las políticas sociales, en los dos municipios pernambucanos estudiados, representan un gran desafío para un sistema político de rasgos peculiares, parecido al de la mayoría de los municipios del interior del Estado. Un número reducido de familias domina los cargos de los poderes ejecutivo y legislativo, y la competencia elec13 Ver un análisis introductorio publicado en LUBAMBO, C. y CIRENO, F. (2009).

14 La investigación en el territorio de Pernambuco fue realizada con la participación de Denílson Bandeira Coelho (UnB). 
toral es fuertemente marcada por las disputas personales, en detrimento de las disputas partidarias. Datos de las cuatro últimas elecciones municipales revelan que un promedio de cinco partidos disputaron las contiendas, y que los candidatos fueron electos con un margen de votos relativamente pequeño, y con ningún partido venciendo en dos ocasiones. Por lo tanto, esos números demuestran que la competitividad política local es alta y la previsibilidad de victoria es baja.

Para los dos casos municipales, (aunque haya una ligera predominancia de la población urbana en el caso de Águas Belas), se destaca la relevancia dada a los proyectos de desarrollo de la actividad rural. Para el acceso al agua o la construcción de cisternas y de unidades sanitarias, las demandas demuestran la necesidad real de sobrevivir de las familias. Mientras que las demandas por educación, salud, transporte y cultura tienen menos relevancia en el contexto local.

Más recientemente, el movimiento social fue fuertemente impulsado en esos y en muchos municipios del territorio, en razón de la exigencia de los programas oficiales de gobierno. Los sindicatos rurales fueron parte importante en la trayectoria de las organizaciones rurales en esos municipios. Varias asociaciones rurales fueron creadas, aún siendo algunas creadas por influencia de los sindicatos. Por causa de la ausencia de Consejos Rurales en el municipio, por ejemplo, asegurar la articulación de proyectos y la participación de las asociaciones es atribución de los Sindicatos de los Trabajadores Rurales

Con la implementación de la nueva política rural del Gobierno Federal y la financiación de proyectos, la estrategia de los sindicatos fue estimular la organización de las asociaciones a partir de núcleos comunitarios. Es notoria la actuación de esas entidades junto a los pequeños productores, incentivándolos a tomar parte de Asociaciones Nucleares de Producción, actividad apoyada por el MDA. Los resultados de esa acción se mostraron bastante positivos, según declaran los entrevistados.

Tal vez en función de ese cuadro, los partidos políticos de Águas Belas fueron definidos por los consejeros del CMDR como entidades “alejadas" de los problemas sociales de la población. El consenso entre los entrevistados es que la organización de la sociedad civil supera la organización de la sociedad política en lo que se refiere a la implementación de políticas públicas sociales. Diversas críticas fueron hechas al comportamiento de los actores políticos locales, los cuales de acuerdo con los consejeros, reivindican de forma aislada el crédito por la aprobación de proyectos para el municipio, al paso que minimizan en ese 
contexto la importancia de las asociaciones. Tal estrategia sería uno de los motivos que explican la evaluación negativa del segmento entre los consejeros del CMDR ${ }^{15}$.

En lo que se refiere a la forma de aplicación de los recursos, la responsabilidad es del Consejo Municipal de Desarrollo Rural. Sin embargo, a pesar del reciente enfrentamiento debido a problemas políticos, suele mantener una relación positiva con el Gobierno Federal y Estadual. Así suele alcanzar éxito en la aprobación y ejecución de proyectos (a ejemplo del Pronaf e PCPR). De un modo general, los consejeros disponen de pocas informaciones sobre la red asociativa rural del territorio. Según ellos no hay cualquier relación entre el CMDR y otros consejos y Fórum Territorial de Desarrollo Rural.

En Paranatama, los factores que determinaron el nuevo modelo participativo estuvieron directamente asociados a dos eventos históricos: el surgimiento del Consejo Municipal de Desarrollo Rural, en 2001, y la aplicación de las reglas formales que demarcan las transacciones entre las asociaciones y el sistema político en el ámbito del propio CMDR. Considerando la evolución desde el primero hasta el segundo momento, en que varias entidades fueron creadas o reorganizadas, se constata que hubo un rompimiento paulatino con el

15 La ausencia de representantes del Ayuntamiento y de la Cámara de Concejales fue recordada por muchos entrevistados. modelo tradicional de participación de tipo clientelista e informal.

El cambio ocurrido, según los entrevistados, fue en función de la institución de un conjunto de 30 nuevas asociaciones comunitarias en el municipio, sumadas a las 15 ya existentes. Sin embargo, no parece que el aumento del número de asociaciones, per se, haya sido la variable que explique el cambio del cuadro en el municipio. La presencia del CMDR sí parece decisiva para la institución de mecanismos de control sobre las políticas públicas. Es notable, por ejemplo, que en el período anterior a la creación del CMDR, no hubo un movimiento social espontáneo en el territorio para la creación de asociaciones o de otras formas de consejos gestores. En ese sentido, claramente el problema no está centrado en el número de asociaciones, pero sí en el tipo de relación con el sistema político de gestión.

\subsection{El Caso de Santa Catarina}

Para el caso de Santa Catarina, el foco del estudio migró de forma forzada de la experiencia asociativa local para la institucionalidad, creada con el Proyecto Mi Sitio. Esto porque no faltan evidencias de que la tradición asociativa de Santa Catarina, de base territorial, fue visiblemente fragilizada después del nuevo modelo de gestión implantado en el Estado. A pesar de reconocidos momentos 
históricos que marcaron el periodo de 1960 a 1996, (movimiento municipal; creación de la Federación Catarinense de Municipios; creación de los Fórums de Desarrollo Regional), el Programa de Descentralización de Santa Catarina, con la institución de las Consejerías de Desarrollo Regional ${ }^{16}$, es el ejemplo paradigmático de cuánto descentralizar puede ser concentrar poder. ${ }^{17}$

El programa en 2003, aunque fuera uno de los ejes estratégicos de la manera en cómo había sido propuesto, no se constituía como instrumento ejecutor de los proyectos de gobierno. La creación de las Consejerías de Desarrollo Regionales, en 2007, en el segundo gobierno de Luís Henrique da Silveira, redimensiona los poderes locales y, a partir de ese momento, el poder se quedó reforzado, realmente regionalizando el Estado. A pesar de la actuación de la Federación Catarinense de Municipios (Fecam), que no dejó de evidenciarse a lo largo del periodo, quedó clara la disminución de su poder. La búsqueda de compatibilización del área geográfica de las SDRs con la de las 21 asociaciones de municipios, (la misma de los fórums/agencias), constituyéndose en una tentativa de inserción en los nuevos planes de descentralización, no fue suficiente para marcar la acción de la Fecam como tradicionalmente se tenía registrado. ${ }^{18}$

16 La Ley LCP - 000243 de 2003 creó 29 SDRs. En 2005, ese número pasó a 30 y, en 2007, se amplió para 36.
En esa perspectiva de análisis, el Proyecto $M i$ Sitio suele ser visto por los entrevistados en general como una tentativa de aumento de poder por parte de las élites. La hipótesis que se asocia a tales constataciones es la del cambio en la configuración de votos regionales, concentrando poder en la mano del grupo político del gobernador.

Con respecto a la participación de la sociedad, se cuestiona la forma de creación que se ha dado, por medio de un decreto de ley idéntico para todas las regiones del estado, independientemente de cada realidad, contrariando los principios de gradualidad descriptos por varios autores. (Desde Lobo (LOBO, 1990). También no atiende al principio de flexibilidad, una vez que no ha considerado las diferencias económico-financieras, políticas, técnico-administrativas y sociales cuando ha implantado un "modelo" único y fijo de descentralización para todas las regiones del estado.

Para Lobo, el conjunto de flexibilidad y de gradualidad tiene como consecuencia el entendimiento de la descentralización como un

y de las redes de cooperación regionales en Santa Catarina para el periodo reciente merece ser ampliada. Merecen destaque los estudios de GAIO (2005) acerca de la experiencia de dos Consejerías de Desarrollo Regional (SDRs); de BIRKNER (2006) sobre el capital social en SC; de ROVER (2007), que hace un análisis de la gestión político-administrativa en tres fórums de desarrollo con actuación en la región Oeste de Santa Catarina; y de CÔRTES (2006) sobre la coordinación horizontal en los gobiernos de los estados Rio Grande do Sul y Santa Catarina. Además, está en curso una investigación acerca de la performance de las SDRs conducida por la Universidad del Estado de Santa Catarina (Udesc), por solicitud del Gobierno del Estado, pero todavía sin resultados publicados. 
proceso en un continuum de tiempo y espacio. Si la centralización se hizo presente en la administración pública brasileña, como resultado de un proceso histórico de formación del Estado, el movimiento contrario sólo podrá ocurrir a partir también de un proceso histórico de alteraciones profundas de ese mismo Estado.

En cuanto a la gestión, en el ámbito de las SDRs, todas las decisiones y acciones son ejecutadas siempre en nombre del Estado, y sus dirigentes son nombrados por el gobernador, sin la participación de fórums o consejos creados regionalmente. Los Consejos de Desarrollo Regional (CDR) podrían ser órganos de importancia fundamental para la consolidación del gobierno en las regiones, una vez que tienen por función definir y deliberar las prioridades, así como servir de instrumento de consulta del gobierno sobre las acciones que serán desarrolladas por las Consejerías de Desarrollo Regional. ${ }^{19}$ Sin embargo, para que el CDR tenga la participación efectiva de los municipios, por medio de los alcaldes y de los presidentes de las cámaras municipales (miembros natos), la representatividad de la comunidad puede ser mejorada. En relación a los demás principios de descentralización

19 Los consejos están subordinados al Consejo Estadual de Desarrollo - Desenvesc, que es presidido por el gobernador del estado e integrado por el vice-gobernador; por los concejales estaduales de Planeamiento; Hacienda; Desarrollo Sostenible; Coordinación y Articulación; así como por el concejal ejecutivo de Articulación Internacional, además de un representante de cada uno de los Consejos de Desarrollo Regional. explorados por Lobo (1988), la transparencia en el proceso de decisión y el control social, aparentemente, son atendidos en el proceso de Santa Catarina por medio de los Consejos de Desarrollo Regional. Para Lobo (1988), la transparencia en el proceso de decisión es fundamental en la descentralización, una vez que redireccionan núcleos de poder hasta entonces localizados centralmente. En ese caso, sin la participación directa de los entes responsables, gobierno estadual y municipal en las etapas de decisión, difícilmente se tendrá el compromiso y la complicidad necesaria para llevar adelante cambios que alteren profundamente las reglas hasta entonces establecidas.

Se ha observado que el proceso de descentralización político-administrativa ocurrido en Santa Catarina, más específicamente en la región de Lages, presenta algunos rasgos de un proceso de desconcentración administrativa, sobre todo por la atribución de ejecición dada a las SDRs. Pero la dependencia financiera, la autonomía limitada para toma de decisiones y la fragilidad en el proceso de participación apuntan en una dirección inversa. Es importante aquí reforzar que la desconcentración es condición necesaria, pero no suficiente para un proceso pleno de descentralización.

Con la visita a la SDR en Lages, quedó evidente lo que algunos actores llaman efectos per- 
versos de políticas públicas. Es posible identificar ciertos ítems contenidos en el diseño institucional del programa de descentralización estadual que, en la fase de implementación, se revelaron como puntos problemáticos $\mathrm{y}$ que pueden 
4. POSIBILIDADES DE CONCEPCIÓN DEL TERRITORIO CON ESTRATEGIA POLÍTICA

La inserción del abordaje territorial envuelve un número extenso de instituciones que, en conjunto, eligen los ejes estratégicos y los proyectos prioritarios del territorio. Aunque las acciones centrales de nivel nacional tengan como agente coordinador la Consejería de Desarrollo Territorial (SDT/MDA), cada estado avanza en ritmo diferenciado, conforme sus programas e institucionalidades. Los dos casos que componen la presente investigación - Pernambuco y Santa Catarina - cuentan con experiencias anteriores de territorialización e incorporan resultados propios para la práctica de gestión en el territorio.

Avances en la percepción del territorio son visibles. Se han discutido, en los fórums de abordaje territorial (bajo cualquier título), temas como el levantamiento de demandas prioritarias para el territorio y de potencialidades estratégicas para acciones colectivas en más de un municipio, sobre todo rurales. En los casos de los dos territorios estaduales estudiados, se han revelado ejemplos exitosos, a pesar de las limitaciones de otras naturalezas ya presentadas.

Sobre el actual diseño institucional de las políticas rurales de desarrollo territorial: ¿Cuál es la percepción de los actores sociales? En las entrevistas realizadas entre los consejeros, se ha evidenciado la falta de conocimiento general sobre el diseño institucional de la política oficial más amplia. Parte de los actores consultados desconocían los objetivos, las líneas de acción y hasta la propia existencia de algunas instancias participativas. Siendo uno de los eslabones de una red territorial, los actores sociales esperan que los fórums actúen de forma referencial para su actuación y articulación política. Pero chocan con una disposición institucional compleja en la que están presentes: en la esfera federal, el Consejo Nacional de Desarrollo Rural Sostenible (Condraf); en la esfera estadual, el Consejo Estadual de Desarrollo Sostenible (CDS); en la esfera territorial, las CIATs y, en la esfera municipal, los actores sociales, que son representados por los CMDRs en Pernambuco y por los Fórums Municipales de Desarrollo Territorial en Santa Catarina. Todos ellos encuentran muchas dificultades para interpretar cuál es la conexión institucional firmada entre las instancias, que son percibidas como un espacio de difícil articulación y dinámica.

Una vez más fue posible comprobar ${ }^{20}$ que, entre los factores que dificultan la actuación de fórums participativos están los conflictos de grupos de intereses/proyectos políticos di-

20 Conclusiones en la misma dirección fueron presentadas por otros autores. Para el caso de Pernambuco, fue publicado un estudio sobre los Consejos de Desarrollo Regionales creados para el Programa Gobierno en los Municipios (LUBAMBO, C. y COÊLHO, D. 2005). Para el caso de Santa Catarina, convergen de forma semejante las conclusiones del estudio de Birkner (BIRKNER, W. 2006). 
vergentes, que se apropian del espacio de las instancias colegiadas. Aunque las discusiones no se orienten a cuestiones partidarias, las soluciones se limitan a estrategias de troca de favores y redistribución de costos y beneficios políticos de los grupos envueltos. Interfiere negativamente también la vinculación de las cuestiones de los antiguos líderes comunitarios, que actúan como representantes de la sociedad civil e incluso gubernamental dentro de las instancias colegiadas. Al final, se refuerza el hecho de que los consejos y fórums son utilizados como espacios de poder para los líderes políticos, (de toda naturaleza), en el territorio.

La desarticulación entre las políticas públicas fue reiteradamente observada en las áreas investigadas tanto en Pernambuco como en Santa Catarina, además de una superposición de acciones; sobre todo en el estado de Santa Catarina. Los técnicos y, muchas veces los gestores, no tienen conocimiento suficiente sobre las otras políticas públicas que actúan en un mismo territorio, desconociendo la actuación, los límites y posibilidades de complementariedad. El hecho de que la población desconoce de un modo general las metodologías de actuación de cada política, sus objetivos y las acciones desarrolladas, contribuye como un elemento a favor de esa situación.

Los desafíos actuales por los cuales pasan los fórums participativos, tanto en lo que dice respecto a la efectividad como en lo que dice respecto a la articulación con otros consejos, tienen raíces en un contexto marcado más por un Estado vaciado que por la ausencia de experiencia asociativa en el territorio. Tal conclusión fue reforzada en varios momentos: a. de un modo general, hubo avances en relación a la participación en los programas rurales/territoriales, en contraposición a la idea de fragilidad asociativa en el campo; b. la participación rural está hoy más institucionalizada; c. aunque no haya garantías de que los programas oficiales de gobierno operen sin interferencia política, es válido afirmar que el papel de las agencias gubernamentales, en el apoyo a las entidades participativas y a la monitorización de ejecución de los proyectos, es fundamental.

Es posible concluir, también, que los grupos pobres de la población rural de Paranatama y Águas Belas, de Campo Belo do Sul, y Cerro Negro carecen de un conjunto de capacidades para influir de forma directa en las políticas públicas de desarrollo territorial. Tales capacidades son, principalmente, de naturaleza organizacional, como la falta de acción colectiva; de naturaleza política, como la falta de capacidad de negociación frente al gobierno; y de naturaleza institucional, como la falta de control sobre las asociaciones y los fórums municipales y territoriales. Esos problemas pueden ser minimizados a partir de la difusión y de la ampliación de la política y, más 
aún, por la continuidad del enfoque participativo.

Retomando los puntos de partida, la investigación ha focalizado los factores políticoinstitucionales que determinan la capacidad de fórums participativos de influenciar en las políticas públicas. Específicamente, considerando el ámbito territorial, el análisis se ha ampliado a la actuación simultánea en niveles distintos, (municipal, territorial, estadual y federal), y ha comprobado las tres suposiciones levantadas inicialmente:

La capacidad de los actores de apropiarse, de forma comprometida, de las instancias de gestión es un factor de gran influencia en el proceso generador y definidor del territorio. Se ha observado que las diversas políticas forman sus territorios a partir de criterios técnicos, no siempre respectando la identidad proferida, cuando el territorio es una construcción social. Una vez que el elemento formador del territorio es el proceso de apropiación de la tierra y de los recursos allí existentes para la sobrevivencia de las personas, el momento de definición de los recortes territoriales no puede prescindir de la participación social. O sea, es evidente la importancia de la red formada por lazos de solidaridad y confianza entre los actores sociales y de la historia del asociativismo territorial.
Una condición central que favorece la articulación horizontal y vertical de los Fórums Participativos es dada por la predisposición de los gestores de las políticas públicas en las tres esferas del poder para el trabajo participativo e integrado. El diálogo entre los gestores de las políticas públicas que actúan en un territorio es la condición siguiente para que haya un ambiente favorable al proceso de articulación en nivel horizontal y vertical de consejos $\mathrm{y}$ fórums participativos $\mathrm{y}$, consecuentemente, una mejor efectividad de eses espacios de participación ciudadana.

La posibilidad de esos consejos y fórums participativos de influenciar y acompañar la implementación de las políticas públicas, en el ejercicio de la gestión territorial, se amplía con la inclusión de un diseño institucional inclusivo. Una vez que se fortalecen las instancias de participación, considerándose la representatividad, se reduce el grado de apropiación de eses espacios por grupos políticos. La adecuada articulación institucional entre las instancias trae en sí un potencial para fortalecer la construcción de cohesión social en torno de proyectos colectivos, en contraposición a la cultura institucional de oferta de políticas públicas negociadas. Ese desafío requiere el ejercicio de nuevos paradigmas que puedan propiciar mayor envolvimiento de las instituciones gubernamentales, de la sociedad civil y de los agentes productivos. 
La lógica sectorial adoptada históricamente por las políticas descentralizadas del Gobierno Federal, contribuye negativamente para la fragmentación en la intervención mediante las políticas públicas. En ese escenario, los actores, aunque participen de diversos consejos simultáneamente, empiezan a desarrollar una visión fragmentada de la realidad en detrimento de una visión global que se compone de esas partes. Es muy común que los consejos no avancen en su misión y sigan figurando simplemente como espacios de reivindicaciones específicas del programa que lo demandó, como fue el caso de los Consejos Municipales de Desarrollo Rural Sostenible, apoyados por el MDA, o de los Fórums Regionales de Desarrollo, creados por los proyectos estaduales.

Por lo expuesto, los desafíos que se colocan delante de la gestión articulada de políticas públicas y de la actuación integrada entre instancias colegiadas en un territorio son grandes. Construir una estrategia de desarrollo territorial en el país depende, entre otros factores, de cambios en el interior de las organizaciones y de los espacios públicos de discusión de desarrollo, sobre todo en el área rural. Estrategias de apoyo al fortalecimiento de esas instancias colegiadas, tales como la implementación de una red de órganos colegiados, municionados de sinergia, identidad y propósitos comunes, amplían la visión para procesos de gestión social, favoreciendo lecturas a partir de los recortes de las comunidades, de los municipios, del territorio y del Estado.

Se abre así un gran espacio para investigaciones futuras. Un análisis sobre el proceso de construcción superpuesta de esas nuevas institucionalidades, así como sobre la cualidad de su acción, es fundamental para que su contribución en el ámbito del desarrollo local sea más efectiva.

\section{REFERENCIAS BIBLIOGRÁFICAS}

Abramovay, R. (2001), Conselhos além dos limites, São Paulo, Estudos Avançados.

Ames, B. (1995), "Electoral Strategy under Open-list Proportional Representation", American Journal of Political Science v. 39, n. 2, pp. 406-433.

Ames, B. (2003), Os entraves da democracia no Brasil, Rio de Janeiro, Editora FGV. 
[ 82 ] Universidad Católica de El Salvador.

Arretche, M. et all (2006), Capital Social, Política Partidária e racionalidade dos atores: estudando os conselhos municipais de desenvolvimento rural comunicación presentada en el $5^{\circ}$ Encuentro de la Asociación Brasileña de Ciencia Política, Belo Horizonte, UFMG.

Birkner, W. M. (2006), Capital Social em Santa Catarina: o caso dos fóruns de desenvolvimento regional, Blumenau, Edifurb.

Carvalho, A. I. (1995), Conselhos de saúde no Brasil: participação cidadã e controle social, Rio de Janeiro, IBAM/FASE.

Coelho, V. y M. Nobre (2004), Participação e Deliberação: Teoria Democrática e Experiências Institucionais no Brasil Contemporâneo, São Paulo, Editora 34.

Côrtes, S. (2006), “Instituições e ação estatal: coordenação horizontal nos governos estaduais catarinense e gaúcho - 2003/2006” en Governo, políticas públicas e elites políticas nos estados brasileiros, Celina Souza, Dantas Neto y Paulo Fábio (Orgs.), Rio de Janeiro, Revan, v. 1, pp. 191-226.

Filippim, E. y F. Abrucio (2008), Quando descentralizar é concentrar poder: a experiência de Santa Catarina, comunicación presentada en el XXXII Enanpad, Rio de Janeiro.

Gaio, A. (2005), As Estratégias de Ação Utilizadas pela Secretaria de Estado de Desenvolvimento Regional de São Joaquim para Implementar o Plano de Desenvolvimento Sustentável de seu Território, disertación presentada en el Máster en Administración de Empresas - Escola Superior de Administração e Gerência - ESAG - UFSC, Florianópolis.

Gohn, M. da G. (2001), Conselhos Gestores e Participação Sócio-politica, São Paulo, Cortez.

GOVERNO DE SANTA CATARINA, disponible en: http://www.sc.gov.br/ Acceso en 28 de enero de 2008. 
Lobo, Thereza (1988), “Descentralização: uma alternativa de mudança” Revista de Administração Pública, Rio de Janeiro v. 22, n 1, pp. 12-33.

Lobo, T. (1990), “Descentralização: conceitos, princípios, prática governamental”, Cadernos de Pesquisa, São Paulo, Fundação Carlos Chagas, nº 74, pp. 5-10.

Löwi, T. (1964), “American Business, Public Policy Case Studies, and Political Theory”, World Politics pp. 677-715, Princeton, University of Princeton Press.

Löwi, T. (1985), “The state in politics: the relation between policy and administration" em Regulatory policy and the social sciences, NOLL, R. (ed.), Berkeley, University of California Press, pp. 67-105.

Lubambo, C. y D. Coelho (2005), Atores Sociais e estratégias de participação no Programa Governo nos Municípios, Petrópolis, Vozes.

Lubambo, C., D. Coelho y M. A. Melo, (Orgs.) (2005), Desenho Institucional e Participação Política: experiências no Brasil contemporâneo, Petrópolis, Vozes.

Lubambo, C. y F. Cireno, (2009), "Participação e Gestão Territorial: onde se encontram as condições favoráveis?”, Revista Cadernos Metrópole, ISSN: 1517-2422, n. 21, pp.197-218.

Melo, M. A. (2003), Empowerment e governança no Brasil: questões conceituais e análise preliminar de experiências selecionadas, Relatório de Pesquisa, Recife, NEPPU/UFPE.

Munarim, A. (2000), Educação e esfera pública na Serra Catarinense: a experiência política do Plano Regional de Educação, Florianópolis, NUP/CEP/UFSC.

Rover, O. J. (2007), Redes de Poder e Governança Local: Análise da gestão político-administrativa em três fóruns de desenvolvimento com atuação na região Oeste de Santa Catarina/Brasil, tesis presentada al Programa de Postgrado en Desarrollo Rural de la Faculdade de Ciências Econômicas, UFRGS. 